\title{
NONCOMMUTATIVE MARKOV PROCESSES $\left({ }^{1}\right)$
}

\author{
BY \\ JOHN DE PILLIS
}

Preview of results. Let $\mathfrak{A}$ be the algebra of linear operators on finite-dimensional Hilbert space $\mathscr{H}$. Let $\eta_{1}$ be a state on $\mathfrak{A}$, i.e., there exists a positive semidefinite (psd) operator $P_{0} \in \mathfrak{A}$ such that $\operatorname{tr}\left(P_{0}\right)=1$ and for all $A \in \mathfrak{A}, \eta_{1}(A)=\operatorname{trace}\left(A P_{0}\right)$. The linear functional $\eta_{n}$ defined on the $n$-fold tensor product $\otimes^{n} \mathfrak{A}$ is defined by setting $\eta_{n}\left(A_{1} \otimes A_{2} \otimes \cdots \otimes A_{n}\right)=\eta_{1}\left(A_{1} \mathbf{T}\left(A_{2} \cdots \mathbf{T}\left(A_{n}\right)\right)\right)$ for $A_{i} \in \mathfrak{A}$, where $\mathbf{T}$ is some linear map sending $\mathfrak{A}$ to itself with the properties that

(i) $\mathbf{T}(A)$ is psd whenever $A$ is psd, and

(ii) $\mathrm{T}(I)=I$, where $I$ is the identity operator on $\mathscr{H} . K_{1}$ will denote the set of all linear maps constrained by properties (i) and (ii).

The theory of Markov processes gives rise to the following noncommutative problem (so-called since the algebra $\mathfrak{A}$ is noncommutative): What relations hold between $\eta_{1}$ and $\mathbf{T}$ in order that the induced linear functional $\eta_{n}$ is, in fact, a state on $\bigotimes^{n} \mathfrak{A}$ ? $\S 1$ contains a more thorough discussion of this noncommutative analog. Some of the results we obtain in answer to this question are the following: If $\mathrm{rg}(\mathbf{T})$, the range of $\mathbf{T}$ is commutative, then $\eta_{2}$ is a state on $\mathfrak{U} \otimes \mathfrak{U}$ if and only if $P_{0}$ commutes with $\mathrm{rg}(\mathrm{T})$ (Theorem 2.3). Corollary 2.9, incidentally, says that it is not unusual that $\mathrm{rg}(\mathrm{T})$ be commutative; in fact, if we suppose that the linear functional $\eta_{n}$ is a bona fide state for all $n$, and if $\eta_{1}(\cdot)=\operatorname{trace}\left(\cdot P_{0}\right)$ where $P_{0}$ has distinct eigenvalues, then $\mathrm{rg}(\mathbf{T})$ is necessarily commutative. We obtain a result which tells us under which circumstances $\eta_{n}$ is a state for all $n$ given only that the functionals $\eta_{1}$ and $\eta_{2}$ are states. In fact, if $\eta_{1}(\cdot)=\operatorname{trace}\left(\cdot P_{0}\right)$, where $P_{0}$ is nonsingular (positive definite), then the functional $\eta_{n}$ is a state on $\otimes^{n} \mathfrak{A}$ for all $n$ if and only if $\eta_{2}$ is a state on $\mathfrak{A} \otimes \mathfrak{A}$ and $\mathrm{rg}(\mathbf{T})$ commutes with $\operatorname{rg}\left(\mathbf{T}^{*}\right)$, where $\mathbf{T}^{*}$ is the Hilbert space adjoint of $\mathbf{T}$ (Theorems 2.6 and 2.7).

It is to be observed that those operators $\mathbf{T} \in K_{1}$ which induce a state $\eta_{2}$ on $\mathfrak{U} \otimes \mathfrak{A}$ is a convex compact set $\mathscr{C}_{\eta_{1}}$, i.e., $\mathscr{C}_{\eta_{1}}=\left\{\mathbf{T}: \mathbf{T} \in K_{1}\right.$ and $\eta_{2}$ is a state on $\mathfrak{A} \otimes \mathfrak{A}\} . \S 3$ is devoted to characterizing the extreme points of $\mathscr{C}_{n_{1}}$ for the case where $\mathfrak{A}$ is the algebra of $2 \times 2$ matrices over the complex field.

1. Introduction. Let $X$ be any abstract set of points $\xi$, and let $I$ be an index set. $\Omega=X^{I}$ will represent the product space whose points $\omega$ are the functions from $I$

Presented to the Society, January 27, 1966; received by the editors February 23, 1966.

${ }^{(1)}$ The results of this paper comprise a portion of the author's doctoral thesis which was written under the direction of Professor Jacob Feldman at the University of California, Berkeley, 1965. The author is gratified to be able to extend his deep appreciation to Professor Feldman, whose suggestions inspired this work. 
to $X$. Suppose we are given a Borel field $\mathscr{F}_{X}$ of sets in $X$. For fixed $i \in I$ and $A \in \mathscr{F}_{X}$ define the set $S(A ; i)$ to be all points $\omega \in \Omega$ such that $\omega(i) \in A . \mathscr{F}$ will denote the Borel field generated by the sets $S(A ; i)$ for $i \in I$ and $A \in \mathscr{F}_{\mathrm{X}}$.

Suppose $p_{0}$ is a probability measure on $\mathscr{F}_{X}$. Suppose $p$ is a function of $(\xi, A)$ where $\xi \in X$ and $A \in \mathscr{F}_{X}$ such that

(i) For fixed $\xi \in X, p(\xi ; \cdot)$ is a probability measure on $\mathscr{F}_{X}$ and

(ii) For fixed $A \in \mathscr{F}_{X}, p(\cdot ; A)$ is measurable with respect to the Borel field $\mathscr{F}_{X}$. Such a $p$ is called a Markov transition function. Utilizing $p_{0}$ and $p$, a probability measure on $\mathscr{F}$ is defined as follows: Let $\Lambda$ be a finite intersection of sets $S(A ; i)$, i.e., $\Lambda=S\left(A_{1} ; i_{1}\right) \cap S\left(A_{2} ; i_{2}\right) \cap \cdots \cap S\left(A_{n} ; i_{n}\right)$. Then

$$
q(\Lambda)=\int_{A_{1}} p_{0}\left(d \xi_{1}\right) \int_{A_{2}} p\left(\xi_{1} ; d \xi_{2}\right) \int_{A_{3}} p\left(\xi_{2} ; d \xi_{3}\right) \cdots \int_{A_{n}} p\left(\xi_{n-1} ; d \xi_{n}\right)
$$

defines a set function $q$ which can be extended to a probability measure on sets in $\mathscr{F}$, where integration is from right to left. This construction is given in a more general setting in Doob [3, p. 613, example 2.6].

In approaching this from a linear functional point of view, suppose $X$ has a topology and is compact so that the topology for $\Omega=X^{I}$ is the product topology. Suppose, too, that $I$ is countable. Let $\mathscr{C}(X)$ denote the algebra of continuous, complex-valued functions on $X$.

We define

$$
f_{1} \otimes f_{2} \otimes \cdots \otimes f_{n} \in \mathscr{C}(\Omega)
$$

by

$$
f_{1} \otimes f_{2} \otimes \cdots \otimes f_{n}(\omega)=f_{1}\left(\omega_{1}\right) f_{2}\left(\omega_{2}\right) \cdots f_{n}\left(\omega_{n}\right)
$$

where each

$$
f_{i} \in \mathscr{C}(X), \quad i=1,2, \ldots, n,
$$

and

$$
\omega=\left(\omega_{1}, \omega_{2}, \ldots, \omega_{n}, \omega_{n+1}, \ldots\right) \in \Omega=X^{I}
$$

Since these functions $f_{1} \otimes f_{2} \otimes \cdots \otimes f_{n}, n=1,2, \ldots$, separate points of $\Omega$, the selfadjoint algebra they generate is uniformly dense in $\mathscr{C}(\Omega)$. If $\mathscr{C}_{n}(\Omega)$ is the subalgebra of $\mathscr{C}(\Omega)$ generated by the functions $f_{1} \otimes f_{2} \otimes \cdots \otimes f_{n}$, then the measure $q$ defined in (1.1) may be identified with the following linear functional $\eta_{(n)}$ on $\mathscr{C}_{n}(\Omega)$.

$$
\begin{aligned}
f_{1} \otimes f_{2} & \otimes \cdots \otimes f_{n} \\
& \stackrel{n_{(n)}}{\longrightarrow} \int_{X} f_{1}\left(\xi_{1}\right) p_{0}\left(d \xi_{1}\right) \int_{X} f_{2}\left(\xi_{2}\right) p\left(\xi_{1} ; d \xi_{2}\right) \cdots \int_{X} f_{n}\left(\xi_{n}\right) p\left(\xi_{n-1} ; d \xi_{n}\right)
\end{aligned}
$$

where integration proceeds from right to left. 
If we define the bounded linear operator $\mathbf{T}$ from $\mathscr{C}(X)$ to $\mathscr{C}(X)$ by

$$
\mathbf{T}(f)\left(\xi_{0}\right)=\int_{X} f(\xi) p\left(\xi_{0} ; d \xi\right) \quad \text { for all } \xi_{0} \in X
$$

then it is to be observed that (1.2) assumes the form

$$
f_{1} \otimes f_{2} \otimes \cdots \otimes f_{n} \stackrel{\eta_{(n)}}{\longrightarrow} \eta_{1}\left(f_{1} \mathbf{T}\left(f_{2} \mathbf{T}\left(\cdots \mathbf{T}\left(f_{n-1} \mathbf{T}\left(f_{n}\right)\right)\right)\right)\right),
$$

where $\eta_{1}$ is the functional on $\mathscr{C}(X), \eta_{1}: f \rightarrow \int_{X} f(\xi) p_{0}(d \xi)$.

We notice that if $f \in \mathscr{C}(X)$ is positive $(f(\xi) \geqq 0$ for all $\xi \in X)$, then so is $\mathbf{T}(f)$, and that $\mathbf{T}\left(1_{x}\right)=1_{x}$ where $1_{x} \in \mathscr{C}(X)$ is the function whose value everywhere on $X$ is one. Moreover, $\eta_{(n)}$ is a state on $\mathscr{C}_{n}(\Omega)$ for every $n=1,2, \ldots$ That is, $\eta_{(n)}(g) \geqq 0$ whenever $g \in \mathscr{C}(\Omega)$ is positive and $\eta_{(n)}\left(1_{\Omega}\right)=1$ where $1_{\Omega} \in \mathscr{C}(\Omega)$ is the function whose value everywhere on $\Omega$ is one, and $\eta_{(n)} / \mathscr{C}_{m}(\Omega)=\eta_{(m)}$ whenever $n>m$.

The general problem that we consider is the following: Replace the commutative algebra $\mathscr{C}(X)$ by a noncommutative algebra, $\mathfrak{A}$, the algebra of linear operators on finite-dimensional Hilbert space, say. In this case, we will require that $\mathbf{T}$ be a linear operator from $\mathfrak{A}$ into itself such that $\mathbf{T}(1)=1$, where 1 is the identity operator in $\mathfrak{A}$, and $\mathbf{T}(A)$ is psd whenever $A$ is psd. Thus, given a state $\eta_{1}$ on the algebra $\mathfrak{A}$, we define the linear functional $\eta_{(n)}$ on $\otimes^{n} \mathfrak{A}$ by

$$
\eta_{(n)}\left(A_{1} \otimes A_{2} \otimes \cdots \otimes A_{n}\right)=\eta_{1}\left(A_{1} \mathbf{T}\left(A_{2} \cdots \mathbf{T}\left(A_{n}\right)\right)\right)
$$

for $A_{i} \in \mathfrak{A}$, which is well defined due to the properties of the tensor product. Our problem concerns the relations between the initial state $\eta_{1}$ and the operator $\mathbf{T}$ which insure that $\eta_{(n)}$ is, in fact, a state on $\otimes^{n} \mathfrak{A}$.

If (1.5) defines $\eta_{(n)}$ as a state, we shall say that $\eta_{1}$ is $n$-extendable by $\mathbf{T}$. If $\eta_{(n)}$ is a state for all $n=1,2,3, \ldots$, then we say simply that $\eta_{1}$ is extendable by $\mathbf{T}$, or T extends $\eta_{1}$. In what follows, $\mathfrak{A}$ will represent a factor of finite type (type $I_{n}$ or $\left.I I_{1}\right)$. In this case, there exists a unique linear functional tr defined on $\mathfrak{A}$ so that

(1) $\operatorname{tr}(1)=1$.

(2) $\operatorname{tr}(\alpha A)=\alpha \operatorname{tr}(A) \quad$ for all scalars $\alpha$, all $A \in \mathfrak{A}$.

(3) $\operatorname{tr}(A+B)=\operatorname{tr}(A)+\operatorname{tr}(B) \quad$ for all $A, B \in \mathfrak{A}$.

(4) $\operatorname{tr}(P) \geqq 0 \quad$ if $P$ is positive definite in $\mathfrak{A}$.

If $P$ is a projection, then $\operatorname{tr}(P)=0 \Rightarrow P=0$.

(5) $\operatorname{tr}(A B)=\operatorname{tr}(B A) \quad$ for all $A, B \in \mathfrak{A}$.

By means of this functional, $\mathfrak{A}$ is given a pre-Hilbert space structure by defining the inner product.

$$
[A, B]=\operatorname{tr}\left(B^{*} A\right) \quad \text { for all } A, B \in \mathfrak{A} .
$$

See Naimark [6, pp. 470, 489]. 
$\otimes^{n} \mathfrak{A}$ inherits a pre-Hilbert space structure, where for

$$
A_{1} \otimes A_{2} \otimes \cdots \otimes A_{n}, B_{1} \otimes B_{2} \otimes \cdots \otimes B_{n} \in \otimes^{n} \mathfrak{A},
$$

we define

(1.8) $\left[A_{1} \otimes A_{2} \otimes \cdots \otimes A_{n}, B_{1} \otimes B_{2} \otimes \cdots \otimes B_{n}\right]=\left[A_{1}, B_{1}\right]\left[A_{2}, B_{2}\right] \cdots\left[A_{n}, B_{n}\right]$.

Extending linearly defines the inner product on all of $\otimes^{n} \mathfrak{A}$. Now suppose $\eta_{1}(\cdot)$ is a countable additive (c.a.) state on $\mathfrak{A}$. From condition (4) of (1.6), $\eta_{1}$ is completely continuous with respect to $\operatorname{tr}(\cdot)$. Hence the structure theorem of I. E. Segal [8] (also H. A. Dye [4, Theorem 4, p. 268]) allows us to write

$$
\eta_{1}(\cdot)=\operatorname{tr}(\cdot P)=[\cdot, P]
$$

where $P$ is a psd operator in $\mathfrak{A}^{c}$, the Hilbert space completion of $\mathfrak{A} . P$ is closed and densely defined.

The question arises; given $P \in \mathfrak{A}$, where $P$ is psd, then which positive-preserving, identity-preserving (bounded) linear operators $\mathbf{T}$ from $\mathfrak{A}$ to $\mathfrak{A}$, extend the state $\eta_{1}(\cdot)=[\cdot, P]$ to a state $\eta_{(n)}(\cdot)$ (see (1.5)) satisfying the following:

$$
\eta_{1}\left(A_{1} \mathbf{T}\left(A_{2} \cdots \mathbf{T}\left(A_{n}\right)\right)\right)=\left[A_{1} \otimes A_{2} \otimes \cdots \otimes A_{n}, Q_{n}\right]
$$

for all $A_{i} \in \mathfrak{A}$ where $Q_{n}$ exists in $\left(\otimes^{n} \mathfrak{A}\right)^{c}$ and is psd?

2. The extendability of $\mathbf{T}$. It is easy to establish conditions which guarantee that $\eta_{(n)}$ is a real linear functional for $n=2$. $\left(\eta_{(n)}\right.$ is real if and only if $\eta_{(n)}(H)$ is real whenever $H=H^{*}$.)

PRoposition 2.1. T induces a real c.a. linear functional $\eta_{2}$ on $\mathfrak{A} \otimes \mathfrak{A}$ if and only if $A \mathbf{T}(B)-\mathbf{T}(B) A$ belongs to the null space of $\eta_{1}$, where $\mathbf{T}: \mathfrak{A} \rightarrow \mathfrak{A}$ is a bounded positive semidefinite-preserving, identity-preserving linear operator, and $\eta_{1}$ is a c.a. state on $\mathfrak{A}$.

Proof. $\eta_{2}$ is real if and only if for all $A, B \in \mathfrak{A}$

$$
\left[\eta_{2}(A \otimes B)\right]^{-}=\eta_{2}\left(A^{*} \otimes B^{*}\right),
$$

where $[\cdot]^{-}$denotes complex conjugation. That is,

$$
\left[\eta_{1}(A T(B))\right]^{-}=\eta_{1}\left(A^{*} T\left(B^{*}\right)\right) \quad \text { for all } A, B \in \mathfrak{A} \text {. }
$$

But $\eta_{1}$ is a state, so that

$$
\left[\eta_{1}(A \mathbf{T}(B))\right]^{-}=\eta_{1}\left((A \mathbf{T}(B))^{*}\right)=\eta_{1}\left(\mathbf{T}\left(B^{*}\right) A^{*}\right) .
$$

Line (2.3) uses the fact that $\mathbf{T}:$ psd $\rightarrow$ psd so that in particular $\mathbf{T}(B)^{*}=\mathbf{T}\left(B^{*}\right)$ for all $B \in \mathfrak{A}$. Subtracting (2.2) from (2.3) proves the result. We are led to a fact which will be of some importance later.

Proposition 2.2. If the induced linear functional $\eta_{2}$ is positive then $\mathrm{rg}(\mathbf{T})$, the range of $\mathrm{T}$, commutes with $P$, where $\eta_{1}(\cdot)=[\cdot, P]$. 
Proof. On one hand,

$$
\begin{aligned}
\eta_{1}(A \mathrm{~T}(B)) & =[A \mathrm{~T}(B), P] \\
& =\left[A, P \mathrm{~T}\left(B^{*}\right)\right]
\end{aligned}
$$

which follows since $[A X, B]=\left[A, B X^{*}\right]$ for all $A, B, X \in \mathfrak{A}$, and since $\mathbf{T}(B)^{*}=$ $\mathbf{T}\left(B^{*}\right)$ for all $B \in \mathfrak{A}$.

On the other hand,

$$
\eta_{1}(A \mathbf{T}(B))=\eta_{2}(A \otimes B) .
$$

Since $\eta_{2}$ is assumed to be a state, $\eta_{2}(A \otimes B) \geqq 0$ whenever $A, B$ are psd in $\mathfrak{A}$. From (2.4) and (2.5) we conclude that

$$
[A, P \mathbf{T}(B)] \geqq 0
$$

if $A$ and $B$ are psd. But this implies that for any fixed psd $B,[\cdot, P \mathbf{T}(B)]$ is a positive functional, which is possible only if $P \mathbf{T}(B)$ is psd. The product of the psd operators $P$ and $\mathbf{T}(B)$ is again psd if and only if they commute, and hence, $P$ and $\mathbf{T}(B)$ commute for all psd $B$. Extending by linearity, we see that $P$ commutes with $\operatorname{rg}(\mathbf{T})$.

In what follows, $\mathfrak{A}$ will be the full algebra of linear operators on finite-dimensional Hilbert space ( $\mathfrak{A}$ is a factor of type $I_{n}$ ).

The next theorem characterizes the states which are 2-extendable by an operator $\mathbf{T}$, provided the range of $\mathbf{T}$ is commutative.

TheOREM 2.3. Let $\mathfrak{A}$ be a factor of type $I_{n}$, and let $\mathbf{T} \in K_{1}$. Assume, moreover, that the range of $\mathbf{T}, \mathrm{rg}(\mathrm{T})$, is commutative. Then the state $\eta_{1}(\cdot)=[\cdot, P]$ is 2-extendable by $\mathbf{T}$ if and only if $P$ commutes with $\mathrm{rg}(\mathbf{T})$, where $P$ is psd in $\mathfrak{A}$.

Proof. If $\eta_{2}(\cdot)$ defined by $\eta_{2}(A \otimes B)=\eta_{1}(A T(B))$ is a state on $\otimes^{2} \mathfrak{A}$, then $P$ commutes with $\operatorname{rg}(\mathbf{T})$ as stated in Proposition 2.2.

Conversely, assume $P$ commutes with $\operatorname{rg}(\mathrm{T})$. For all $B \in \mathfrak{A}$, we note that

$$
\mathbf{T}(B)=\sum\left[B, P_{i}\right] P_{x_{i}}
$$

where each $P_{i}$ is psd in $\mathfrak{A}, \operatorname{tr}\left(P_{i}\right)=1, i=1,2, \ldots, n,\left\{P_{x_{1}}, P_{x_{2}}, \ldots, P_{x_{n}}\right\}$ is a spectral family of orthogonal one-dimensional projections for $\operatorname{rg}(\mathbf{T})$, which is commutative. Now

$$
\begin{aligned}
\eta_{1}(A \mathbf{T}(B)) & =[A \mathbf{T}(B), P] \\
& =\left[A, P \mathbf{T}\left(B^{*}\right)\right] \\
& =\sum_{i=1}^{n}\left[A, P\left[B^{*}, P_{i}\right] P_{x_{i}}\right] \\
& =\sum_{i=1}^{n}\left[A, P P_{x_{i}}\right]\left[B, P_{i}\right] \\
& =\left[A \otimes B, \sum_{i=1}^{n} P P_{x_{i}} \otimes P_{i}\right] .
\end{aligned}
$$


Since $P$ is assumed to commute with $\operatorname{rg}(\mathrm{T}), P$ necessarily commutes with the spectral family $\left\{P_{x_{1}}, P_{x_{2}}, \ldots, P_{x_{n}}\right\}$. Thus, $P \otimes 1$ commutes with $P_{x_{i}} \otimes P_{i}$ for all $i=1,2, \ldots, n$, and the product of commuting psd operators, $P P_{x_{i}} \otimes P_{i}$ is again psd. We conclude that $\sum_{i=1}^{n} P P_{x_{i}} \otimes P_{i}$ is psd in $\otimes^{2} \mathfrak{A}$. Since $\operatorname{tr}\left(\sum_{i=1}^{n} P P_{x_{i}} \otimes P_{i}\right)=1$,

$$
\eta_{1}(A \mathbf{T}(B))=\left[A \otimes B, \sum_{i=1}^{n} P P_{x_{i}} \otimes P_{i}\right]=\eta_{2}(A \otimes B)
$$

defines the linear functional $\eta_{2}$ as a state on $\otimes^{2} \mathfrak{A}$, and the theorem is proved.

So far we have concerned ourselves with the question of 2-extendability of the c.a. state $\eta_{1}(\cdot)$ by the operator T. Our next theorem demonstrates that if $\mathrm{rg}(\mathbf{T})$ commutes with $\mathrm{rg}\left(\mathbf{T}^{*}\right)$, then 2-extendability is sufficient to assure $n$-extendability of a state $\eta_{1}(\cdot)$ for $n=1,2,3, \ldots$

After this theorem is proved, we will prove the converse in that if the state $\eta_{1}(\cdot)=[\cdot, P]$, and the psd operator $P$ has an inverse $P^{-1}$, then every operator $\mathbf{T}$ which extends the state $\eta_{1}(\cdot)$ for all $n=1,2,3, \ldots$ has the property that $\operatorname{rg}(\mathbf{T})$ commutes with $\operatorname{rg}\left(\mathbf{T}^{*}\right)$.

First we prove

LEMMA 2.4. If $\mathbf{T}$, a bounded linear operator from $\mathfrak{A}$ into $\mathfrak{A}$, commutes with the adjoint operation, then $\mathscr{J}(T)=\mathscr{J}\left(T^{*}\right)^{\circ}$ where $\mathscr{J}$ is defined by

$$
[\mathscr{J}(T), A \otimes B]=\left[\mathrm{T}\left(A^{*}\right) ; B\right]
$$

for all $A, B \in \mathfrak{A}$ and $X^{\circ}$ is defined for every $X \in \otimes^{2} \mathfrak{A}$ by

$$
\left[X^{\circ}, A \otimes B\right]=[X, B \otimes A]
$$

for all $A, B \in \mathfrak{A}$.

Proof.

$$
\begin{aligned}
{\left[\mathscr{J}(\mathrm{T}), A^{*} \otimes B\right] } & =\left[\mathbf{T}\left(A^{*}\right), B\right]=\left[A^{*}, \mathbf{T}^{*}(B)\right] \\
& =\left[\mathbf{T}^{*}\left(B^{*}\right), A\right]=\left[\mathscr{J}\left(\mathbf{T}^{*}\right), B \otimes A\right] \\
& =\left[\mathscr{J}\left(\mathbf{T}^{*}\right)^{o}, A \otimes B\right]
\end{aligned}
$$

for all $A, B \in \mathfrak{A}$. Therefore, $\mathscr{J}(\mathbf{T})=\mathscr{J}\left(\mathbf{T}^{*}\right)^{\circ}$, and the lemma is proved.

LEMMA 2.5. For $\mathbf{T}$ a bounded linear operator from $\mathfrak{A}$ into $\mathfrak{A}$ which commutes with the adjoint operation, $\mathrm{rg}(\mathbf{T})$ commutes with $\mathrm{rg}\left(\mathbf{T}^{*}\right)$ if and only if $\mathscr{J}(\mathbf{T}) \otimes 1$ commutes with $1 \otimes \mathscr{J}(\mathbf{T})$.

Proof. Note that $\mathscr{J}(\mathbf{T}) \in \bigotimes^{2} \mathfrak{A}$, and that 1 is the identity operator of $\mathfrak{A}$.

There are two identities $\left(\left(2.10^{\prime}\right)\right.$ and $\left.\left(2.11^{\prime \prime}\right)\right)$ we will need. We define an "opposite operator" $Q$ on $\otimes^{3} \mathfrak{A}$, (to be distinguished from the operator ${ }^{\circ}$ on $\otimes^{2} \mathfrak{A}$ previously defined) by

$$
\left[X^{Q}, A \otimes B \otimes C\right]=[X, C \otimes B \otimes A]
$$


for all $X \in \otimes^{3} \mathfrak{A}$ and all $A, B, C \in \mathfrak{A}$. That is

$(A \otimes B \otimes C)^{\circ}=C \otimes B \otimes A$.

A relation between the operators $Q$ and ${ }^{\circ}$ is given by

$\left(\left(A_{1} \otimes A_{2} \otimes 1\right) \cdot\left(1 \otimes B_{1} \otimes B_{2}\right)\right)^{\circ}$

$$
=B_{2} \otimes A_{2} B_{1} \otimes A_{1}
$$

$$
\begin{aligned}
& =\left(1 \otimes A_{2} \otimes A_{1}\right)\left(B_{2} \otimes B_{1} \otimes 1\right) \\
& =1 \otimes\left(A_{1} \otimes A_{2}\right)^{\circ} \cdot\left(B_{1} \otimes B_{2}\right)^{\circ} \otimes 1 .
\end{aligned}
$$

Extension by linearity tells us that for all $X \in Y \in \bigotimes^{2} \mathfrak{A}, 1 \in \mathfrak{A}$,

$$
((X \otimes 1) \cdot(1 \otimes Y))^{\circ}=\left(1 \otimes X^{\circ}\right)\left(Y^{\circ} \otimes 1\right) .
$$

We consider the other identity. For any state $\eta_{1}(\cdot)=[\cdot, P]$,

$$
\begin{aligned}
\eta_{1}(A \mathbf{T}(B)) & =[A \mathbf{T}(B), P] \\
& =\left[\mathbf{T}(B), A^{*} P\right] \\
& =\left[\mathscr{J}(\mathbf{T}), B^{*} \otimes A^{*} P\right] \\
& =\left[\mathscr{J}(\mathbf{T})(1 \otimes P), B^{*} \otimes A^{*}\right] \\
& =[B \otimes A,(1 \otimes P) \cdot \mathscr{J}(\mathbf{T})] \text { since } \mathscr{J}(\mathbf{T})=\mathscr{J}(\mathbf{T})^{*} \\
& =\left[A \otimes B,\{(1 \otimes P) \cdot \mathscr{J}(\mathbf{T})\}^{\circ}\right] \\
& =\left[A \otimes B,(P \otimes 1) \mathscr{J}(\mathbf{T})^{\circ}\right] .
\end{aligned}
$$

Now for $B$, we substitute $B \mathrm{~T}(C)$. Hence, (2.11) becomes

$$
\eta_{1}(A \mathrm{~T}(B \mathrm{~T}(C)))=\left[A \otimes B \mathrm{~T}(C),(P \otimes 1) \mathscr{J}(\mathbf{T})^{\circ}\right] .
$$

Now if $\left\{E_{i}\right\} i=1,2, \ldots, n^{2}$, is a basis for $\mathfrak{A}$, then $\left\{E_{i} \otimes E_{j}\right\}, i, j=1,2, \ldots, n^{2}$ forms a basis for $\otimes^{2} \mathfrak{A}$. Let $\mathscr{J}(\mathbf{T})=\sum r_{i j}\left(E_{i} \otimes E_{j}\right) i, j=1,2, \ldots, n^{2}$. Then $\left(2.11^{\prime}\right)$ becomes

$$
\begin{aligned}
\eta_{1}(A \mathrm{~T}(B T(C))) \\
\quad=\sum_{i, j=1}^{n^{2}} r_{i j}[A \otimes B \mathrm{~T}(C)]\left[P E_{j} \otimes E_{i}\right] \\
\quad=\sum_{i, j=1}^{n^{2}} r_{i j}\left[A, P E_{j}\right]\left[B \mathrm{~T}(C), E_{i}\right] \\
\quad=\sum_{i, j=1}^{n^{2}} r_{i j}\left[A, P E_{j}\right]\left[\mathrm{T}(C), B^{*} E_{i}\right] \\
=\sum_{i, j=1}^{n^{2}} r_{i j}\left[A, P E_{j}\right] \cdot\left[\mathscr{J}(\mathrm{T}), C^{*} \otimes B^{*} E_{i}\right] \\
=\sum_{i, j=1}^{n^{2}} r_{i j}\left[A, P E_{j}\right]\left[C \otimes E_{i}^{*} B, \mathscr{J}(\mathrm{T})\right]
\end{aligned}
$$




$$
\begin{aligned}
& =\sum_{i, j=1}^{n^{2}} r_{i j}\left[A, P E_{j}\right]\left[C \otimes B,\left(1 \otimes E_{i}\right) \mathscr{J}(\mathbf{T})\right] \\
& =\sum_{i, j=1}^{n^{2}} r_{i j}\left[A, P E_{j}\right]\left[B \otimes C,\left(E_{i} \otimes 1\right) \mathscr{J}(\mathrm{T})^{\circ}\right]
\end{aligned}
$$
continued

$$
\begin{aligned}
& =\left[A \otimes B \otimes C, \sum_{i, j=1}^{n^{2}} r_{i j} P E_{j} \otimes\left[\left(E_{i} \otimes 1\right) \mathscr{J}(\mathbf{T})^{\circ}\right]\right] \\
& =\left[A \otimes B \otimes C,(P \otimes 1 \otimes 1)\left(\sum_{i, j=1}^{n^{2}} r_{i j} E_{j} \otimes E_{i} \otimes 1\right)\left(1 \otimes \mathscr{J}(\mathbf{T})^{\circ}\right)\right] \\
& =\left[A \otimes B \otimes C,(P \otimes 1 \otimes 1)\left(\mathscr{J}(\mathbf{T})^{\circ} \otimes 1\right)\left(1 \otimes \mathscr{J}(\mathbf{T})^{\circ}\right)\right] .
\end{aligned}
$$

Now $\operatorname{rg}(\mathbf{T})$ commutes with $\operatorname{rg}\left(\mathbf{T}^{*}\right)$ if and only if

$$
\left[B, \mathbf{T}^{*}(A) \mathbf{T}(C)\right]=\left[B, \mathbf{T}(C) \mathbf{T}^{*}(A)\right] \quad \text { for all } A, B, C \in \mathfrak{A} .
$$

But the right side of (2.12) can be written

$$
\begin{aligned}
{\left[B, \mathbf{T}(C) \mathbf{T}^{*}(A)\right] } & =\left[B \mathrm{~T}^{*}\left(A^{*}\right), \mathrm{T}(C)\right] \\
& =\left[\mathrm{T}^{*}\left(B \mathrm{~T}^{*}\left(A^{*}\right)\right), C\right] \\
& =\left[C^{*} \mathbf{T}^{*}\left(B \mathrm{~T}^{*}\left(A^{*}\right)\right), 1\right]
\end{aligned}
$$

Using $\left(2.11^{\prime \prime}\right)$ we obtain

$$
\begin{aligned}
\eta_{1}\left(C^{*} \mathbf{T}^{*}\left(B \mathbf{T}^{*}\left(A^{*}\right)\right)\right) & =\left[C^{*} \mathbf{T}^{*}\left(B \mathbf{T}^{*}\left(A^{*}\right)\right), 1\right], \\
& =\left[C^{*} \otimes B \otimes A^{*},\left(\mathscr{J}\left(\mathbf{T}^{*}\right)^{\circ} \otimes 1\right)\left(1 \otimes \mathscr{J}\left(\mathbf{T}^{*}\right)^{\circ}\right)\right],
\end{aligned}
$$

and by Lemma 2.4 , this

$$
=\left[C^{*} \otimes B \otimes A^{*},(\mathscr{J}(\mathbf{T}) \otimes 1)(1 \otimes \mathscr{J}(\mathbf{T}))\right] .
$$

On the other hand, the left side of (2.12) becomes

$$
\begin{aligned}
{\left[B, \mathbf{T}^{*}(A) \mathbf{T}(C)\right] } & =\left[B \mathbf{T}\left(C^{*}\right), \mathbf{T}^{*}(A)\right] \\
& =\left[\mathbf{T}\left(B \mathbf{T}\left(C^{*}\right)\right), A\right] \\
& =\left[A^{*} \mathbf{T}\left(B \mathbf{T}\left(C^{*}\right)\right), 1\right]
\end{aligned}
$$

Appealing once again to $\left(2.11^{\prime \prime}\right)$,

$$
\begin{aligned}
& =\left[A^{*} \otimes B \otimes C^{*},\left(\mathscr{J}(\mathbf{T})^{\circ} \otimes 1\right)\left(1 \otimes \mathscr{J}(\mathbf{T})^{\circ}\right)\right] \\
& =\left[C^{*} \otimes B \otimes A^{*},\left\{\left(\mathscr{J}(\mathbf{T})^{\circ} \otimes 1\right)\left(1 \otimes \mathscr{J}(\mathbf{T})^{\circ}\right\}^{\circ}\right] .\right.
\end{aligned}
$$


Using (2.10')

$$
=\left[C^{*} \otimes B \otimes A^{*},(1 \otimes \mathscr{J}(\mathbf{T})) \cdot(\mathscr{F}(\mathbf{T}) \otimes 1)\right]
$$

for all $A, B, C \in \mathfrak{A}$. Comparing (2.12a) with (2.12b) we conclude that $\operatorname{rg}(\mathrm{T})$ commutes with $\operatorname{rg}\left(T^{*}\right)$ if and only if

$$
(1 \otimes \mathscr{J}(\mathbf{T}))(\mathscr{J}(\mathbf{T}) \otimes 1)=(\mathscr{J}(\mathbf{T}) \otimes 1)(1 \otimes \mathscr{J}(\mathbf{T})) ;
$$

that is, if and only if $1 \otimes \mathscr{J}(\mathbf{T})$ commutes with $\mathscr{J}(\mathbf{T}) \otimes 1$ and the lemma is proved.

We come to the theorem which gives a sufficient condition for 2-extendability of a state $\eta_{1}(\cdot)$ to imply $n$-extendability of $\eta_{1}(\cdot)$, for all $n$.

THEOREM 2.6. Suppose $\mathbf{T}$ is a linear operator from $\mathfrak{A}$ to $\mathfrak{A}$ where $T \in K_{1}$. Suppose $\mathrm{rg}(\mathbf{T})$ commutes with $\mathrm{rg}\left(\mathbf{T}^{*}\right)$ and that a state $\eta_{1}(\cdot)$ on $\mathfrak{A}$ is 2-extendable by $\mathbf{T}$. Then $\eta_{1}(\cdot)$ is $n$-extendable by $\mathbf{T}$ for all $n=1,2,3, \ldots$ whenever $\eta_{1}(\cdot)=[\cdot, P]$ and $P^{-1}$ exists.

Proof. An inductive argument which imitates the demonstration of (2.11") yields

$$
\begin{aligned}
& \eta_{1}\left(A_{1} \mathbf{T}\left(A_{2} \cdots \mathbf{T}\left(A_{n}\right)\right)\right) \\
& \quad=\left[A_{1} \otimes A_{2} \otimes \cdots \otimes A_{n},(P \otimes 1 \otimes \cdots \otimes 1)\left(\mathscr{J}(\mathbf{T})^{\circ} \otimes 1 \otimes \cdots \otimes 1\right)\right. \\
& \left.\cdots\left(1 \otimes \cdots \otimes \mathscr{J}(\mathbf{T})^{\circ}\right)\right]
\end{aligned}
$$

where $\eta_{1}(\cdot)=[\cdot, P]$ defines $P \in \mathfrak{A}$.

Now (2.13) defines a state on $\otimes^{n} \mathfrak{A}$ if and only if

$$
\begin{aligned}
(P \otimes 1 \otimes \cdots \otimes 1)\left(\mathscr{J}(\mathbf{T})^{\circ} \otimes I \otimes \cdots \otimes 1\right)\left(1 \otimes \mathscr{J}(\mathbf{T})^{\circ} \otimes \cdots \otimes 1\right) \\
\cdots\left(1 \otimes \cdots \otimes 1 \otimes \mathscr{J}(\mathbf{T})^{\circ}\right)
\end{aligned}
$$

is psd in $\otimes^{n} \mathfrak{A}$. We have assumed that $\eta_{1}(\cdot)$ is 2-extendable so that we already have, as a special case of (2.14),

$$
(P \otimes 1) \cdot \mathscr{J}(\mathbf{T})^{\circ} \quad \text { is psd in } \otimes^{2} \mathfrak{A} .
$$

Since $(P \otimes 1)^{-1}$ exists and is psd, and since $\mathscr{J}(\mathbf{T})^{\circ}=\mathscr{J}(\mathbf{T})^{\circ *},(2.15)$ obtains if and only if $\mathscr{J}(\mathbf{T})^{\circ}$ is psd also. To see this suppose $P$ is psd and $H=H^{*}$. Then $P H=(P H)^{*}=H P=Q$ which is psd, and this leads to $H=P^{-1} Q=Q P^{-1}$. That is, $Q$, which is psd, commutes with the psd $P^{-1}$, which is equivalent to saying that the product, $H$, is psd. Thus every factor in (2.14) is psd. Since $\mathrm{rg}(\mathrm{T})$ commutes with $\mathrm{rg}\left(\mathrm{T}^{*}\right)$, adjacent factors commute (Lemma 2.5). In any case, nonadjacent factors will always commute. Thus, the product of commuting psd operators is psd, which applies to (2.14), and the theorem is proved.

The converse, which follows more easily, is now given. 
THEOREM 2.7. If the state $\eta_{1}(\cdot)=[\cdot, P]$ where $P^{-1}$ exists, and if $\eta_{1}(\cdot)$ is n-extendable by $\mathbf{T}$ for $\mathbf{T}$ a bounded linear operator from $\mathfrak{A}$ to $\mathfrak{A}, \mathbf{T} \in K_{1}$, then necessarily, $\mathrm{rg}(\mathrm{T})$ commutes with $\mathrm{rg}\left(\mathrm{T}^{*}\right)$.

Proof. A proof can be extracted from the techniques of the previous proof using (2.13) and Lemma 2.5, but a brief, direct proof exists, viz.

$$
\begin{aligned}
\eta_{3}(A \otimes B \otimes C) & =\eta_{1}(A \mathrm{~T}(B \mathrm{~T}(C))) \\
& =[A \mathbf{T}(B \mathbf{T}(C)), P] \\
& =\left[\mathbf{T}(B \mathbf{T}(C)), A^{*} P\right] \\
& =\left[B \mathbf{T}(C), \mathbf{T}^{*}\left(A^{*} P\right)\right] \\
& =\left[B, \mathbf{T}^{*}\left(A^{*} P\right) \mathbf{T}\left(C^{*}\right)\right] .
\end{aligned}
$$

Now $\eta_{3}$ is assumed to be a state; also, whenever $A, B, C$ are psd, then so is $A \otimes B \otimes C$. Hence, (2.16) is nonnegative under these circumstances. That is, for all $A, B, C$, psd,

$$
\left[B, \mathbf{T}^{*}\left(A^{*} P\right) \mathbf{T}\left(C^{*}\right)\right] \geqq 0 .
$$

Since $B$ runs over all psd operators, we conclude

$$
\mathbf{T}^{*}\left(A^{*} P\right) \mathbf{T}\left(C^{*}\right) \text { is psd whenever } A, C \text { are psd. }
$$

Since $\mathbf{T} \in K_{1}$ we know that $\mathbf{T}\left(C^{*}\right)$ is psd for $C^{*}$ psd. Setting $C^{*}=1$, (2.18) implies

$$
\mathrm{T}^{*}\left(A^{*} P\right) \text { is psd for every psd } A .
$$

Thus, (2.18), which is the product of two psd operators, is again psd if and only if the terms $\mathrm{T}^{*}\left(A^{*} P\right)$ and $\mathrm{T}\left(C^{*}\right)$ commute for all $A, C$ psd in $\mathfrak{A}$. By linear extension, $\mathrm{T}^{*}(A P)$ commutes with $\mathrm{T}(C)$ for all $A, C \in \mathfrak{A}$. Replacing $A$ with $A P^{-1}$, yields $\mathbf{T}^{*}(A)$ commutes with $\mathbf{T}(C)$ for all $A, C \in \mathfrak{A}$, which was to be proved.

Having established necessary and sufficient conditions for certain states $\eta_{1}$ to be $n$-extendable by certain operators $\mathbf{T}$, we proceed to define the fully extended algebra $\otimes^{\infty} \mathfrak{A}$ with its fully extended state $\eta_{\infty}$.

For all $n=1,2,3, \ldots$, we shall identify $\otimes^{n} \mathfrak{A}$ with a subalgebra of $\otimes^{n+1} \mathfrak{A}$ by the following map for all $A_{1}, A_{2}, \ldots, A_{n} \in \mathfrak{A}$;

$$
A_{1} \otimes A_{2} \otimes \cdots \otimes A_{n} \rightarrow A_{1} \otimes A_{2} \otimes \cdots \otimes A_{n} \otimes 1,
$$

which, when extended by linearity, imbeds $\otimes^{n} \mathfrak{A}$ into $\otimes^{n+1} \mathfrak{A}$. Thus we have the ordered relation $\mathfrak{U} \subset \otimes^{2} \mathfrak{U} \subset \otimes^{3} \mathfrak{U} \subset \ldots$ and so it is meaningful to define $\otimes^{\infty} \mathfrak{U}=\bigcup_{n=1}^{\infty} \otimes^{n} \mathfrak{A}$.

Similarly, $\eta_{(n)}$ considered as a set of ordered pairs is contained in $\eta_{(n+1)}$ since the state $\eta_{(n+1)}$, when restricted to $\otimes^{n} \mathfrak{A}$, agrees with $\eta_{(n)}$. Hence $\eta_{\infty}=\bigcup_{n=1}^{\infty} \eta_{n}$ is defined on $\otimes^{\infty} \mathfrak{A}$. From (2.13) and (2.14), we see that $\eta_{\infty}$ is a state on $\otimes^{\infty} \mathfrak{A}$. 
Let $\mathfrak{A}_{n}$ be the algebra of linear operators on the $n$-dimensional Hilbert space $\mathscr{H}_{\text {. }}$. Suppose the state $\eta_{1}(\cdot)=[\cdot, P]$ is defined on $\mathfrak{A}_{n}$ where $P$ is psd in $\mathfrak{A}_{n}$ and $\operatorname{tr}(P)=1$. The spectral decomposition of $P$ allows us to represent $P$ by

$$
P=\sum_{i=1}^{k} \lambda_{i} \mathscr{M}_{i} \quad \text { where } \lambda_{1}>\lambda_{2}>\cdots>\lambda_{k} \geqq 0,
$$

and $\mathscr{M}_{i}$ is a subspace of $\mathscr{H}_{n}$ where $\operatorname{dim}\left(\mathscr{M}_{i}\right)=n_{i}$, and $\mathscr{H}_{n}=\mathscr{M}_{1} \oplus \mathscr{M}_{2} \oplus \cdots \oplus \mathscr{M}_{k}$. Since the $\lambda_{i}$ 's are distinct, the only operators $A$ which commute with $P$ are of the form

$$
A=A_{1} \oplus A_{2} \oplus \cdots \oplus A_{k}
$$

where $A_{i}$ is a linear operator from $\mathscr{M}_{i}$ into $\mathscr{M}_{i}$ (an $n_{i} \times n_{i}$ matrix). Equivalently stated, $\mathscr{M}_{i} A \mathscr{M}_{j}=A_{i} \mathscr{M}_{i} \mathscr{M}_{j}$ for all $i, j=1,2, \ldots, k$ if and only if $A$ commutes with $P=\sum^{k} \lambda_{i} \mathscr{M}_{i}$. By Proposition 4.2, and (2.21), we arrive at

Proposition 2.8. Suppose $\mathbf{T}$ is linear from $\mathfrak{A}_{n}$ into $\mathfrak{A}_{n}$ and that $\mathbf{T} \in K_{1}$. If $\mathbf{T}$ extends the state $\eta_{1}(\cdot)=[\cdot, P]$, then for all $A \in \mathfrak{A}_{n}$,

$$
\mathbf{T}(A)=\mathbf{T}_{1}(A) \oplus \mathbf{T}_{2}(A) \oplus \cdots \oplus \mathbf{T}_{k}(A)
$$

where

$$
P=\sum_{i=1}^{k} \lambda_{i} \mathscr{M}_{i}, \quad \lambda_{1}>\lambda_{2}>\cdots>\lambda_{k} \geqq 0
$$

and $\mathbf{T}_{i}(A)$ is an operator from $\mathscr{M}_{i}$ into $\mathscr{M}_{i}$.

There are a number of observations to be made as to the implications of Proposition 2.8. Since $\mathbf{T} \in K_{1}$, each $\mathbf{T}_{i}(A)$ must be psd whenever $A$ is psd. Thus, $\mathbf{T}_{i}$ as defined by (2.22), is an operator from $\mathfrak{A}_{n}$ into $\mathfrak{A}_{n_{i}}$ where $\mathbf{T}_{i}\left(1_{n}\right)=1_{n_{i}}$ and $\mathbf{T}_{i} \in K_{1_{n_{i}}}, 1_{n_{i}}$ is the identity of $\mathfrak{A}_{n_{i}}$.

Statement (2.22) does not say that $\mathbf{T}$ itself is a direct sum of operators, but the range of $\mathbf{T}$ can be thought of as a direct sum. An interesting and easily obtained corollary follows.

COROllary 2.9. Suppose $\eta_{1}(\cdot)=[\cdot, P]$ is a state on $\mathfrak{A}_{n}$. The only operators $\mathbf{T}$ which extend $\eta_{1}(\cdot)$, necessarily have commutative range, where $\mathbf{T} \in K_{1}$, if it is assumed that the eigenvalues of $P$ are distinct (of multiplicity one).

Proof. Since $P=\sum_{i=1}^{n} \lambda_{i} P_{x_{i}},(2.22)$ implies that $\mathbf{T}(A)=\sum_{i=1}^{n} \mathbf{T}_{i}(A) \cdot P_{x_{i}}$ where $\mathbf{T}_{i}$ is a state on $\mathfrak{A}_{n}$. I.e., $\mathbf{T}(A)=\sum_{i=1}^{n}\left[A, P_{i}\right] P_{x_{i}}$ where $P_{i}$ is psd of trace one, and $\sum P_{x_{i}}=1$.

The decomposition of $\mathrm{rg}(\mathrm{T})$ as given by Proposition 2.8 gives us a means of establishing the structure of $\mathscr{J}(\mathbf{T})$, where $[\mathbf{T}(A), B]=\left[\mathscr{J}(\mathbf{T}), A^{*} \otimes B\right]$ by definition. 
THEOREM 2.10. Let $\eta_{1}(\cdot)=[\cdot, P]$ be a state on $\mathfrak{A}_{n}$ and let $\mathbf{T}$ be a linear operator from $\mathfrak{A}_{n}$ into itself which commutes with the adjoint. Suppose $P=\sum_{i=1}^{k} \lambda_{i} \mathscr{M}_{i}$ where $\lambda_{1}>\lambda_{2}>\cdots>\lambda_{k}>0$. If $\eta_{1}(A \mathrm{~T}(B))=[A \otimes B, Q]$ for all $Q \in \otimes^{2} \mathfrak{A}$, then

$$
Q_{2}=\mathscr{J}_{1}\left(\mathbf{T}_{1}\right)^{\circ} \otimes \mathscr{J}_{2}\left(\mathbf{T}_{2}\right)^{\circ} \otimes \cdots \otimes \mathscr{J}_{k}\left(\mathbf{T}_{k}\right)^{\circ}
$$

where $\mathbf{T}_{i}$ is defined in Proposition 2.8 and $\left[\mathbf{T}_{i}(A), B\right]=\left[\mathscr{J}_{i}\left(\mathbf{T}_{i}\right), A^{*} \otimes B\right]$ defines $\mathscr{J}_{i}$ for all $A \in \mathfrak{A}_{n}, B \in \mathfrak{A}_{n_{1}}$. Hence, for $\lambda_{1}>\lambda_{2}>\cdots>\lambda_{k}>0, Q_{2}$ is psd (T 2-extends $\eta$ ) implies that $\mathscr{J}_{1}\left(\mathbf{T}_{1}\right)^{\circ}, \mathscr{J}_{2}\left(\mathbf{T}_{2}\right)^{\circ}, \ldots, \mathscr{J}_{k}\left(\mathbf{T}_{k}\right)^{\circ}$ are psd.

\section{Proof.}

$$
\begin{aligned}
\eta_{1}(A \mathbf{T}(B)) & =\left[A \mathbf{T}(B), \sum \lambda_{i} \mathscr{M}_{i}\right] \\
& =\sum_{i p q r s}\left[\mathscr{M}_{p} A \mathscr{M}_{q} \cdot \mathscr{M}_{r} \mathbf{T}(B) \mathscr{M}_{s}, \lambda_{i} \mathscr{M}_{i}\right] \\
& =\sum_{i} \lambda_{i}\left[\mathscr{M}_{i} A \mathscr{M}_{i} \cdot \mathscr{M}_{i} \mathbf{T}(B) \mathscr{M}_{i}, \mathscr{M}_{i}\right] \\
& =\sum_{i} \lambda_{i}\left[A_{i i} ; \mathbf{T}_{i}(B), \mathscr{M}_{i}\right]
\end{aligned}
$$

where $A_{i j}=\mathscr{M}_{i} A \mathscr{M}_{j}, \mathbf{T}_{i}(B)=\mathscr{M}_{i} \mathbf{T}(B) \mathscr{M}_{i}$ by definition

$$
\begin{aligned}
& =\sum \lambda_{i}\left[\mathbf{T}_{i}(B), A_{i i}^{*}\right] \\
& =\sum \lambda_{i}\left[\mathscr{J}_{i}\left(\mathbf{T}_{i}\right), B^{*} \otimes A_{i i}^{*}\right] \\
& =\sum \lambda_{i}\left[B \otimes A_{i i}^{*}, \mathscr{J}_{i}\left(\mathbf{T}_{i}\right)\right] \\
& =\sum \lambda_{i}\left[A_{i i} \otimes B, \mathscr{J}_{i}\left(\mathbf{T}_{i}\right)^{o}\right]=[A \otimes B, Q]
\end{aligned}
$$

by hypothesis. Now if for $A, \mathscr{M}_{p} A \mathscr{M}_{q}$ is substituted into the second line of the proof, we see that $\left[\mathscr{M}_{p} A \mathscr{M}_{q} \otimes B, Q\right]=0$ for all $A, B \in \mathfrak{A}_{n}$ if $p \neq q$. That is, the only $A \otimes B$ which do not go to zero are of the form

$$
\left(A_{11} \otimes A_{22} \otimes \cdots \otimes A_{k k}\right) \otimes B=\sum E_{i i} \otimes A_{i i} \otimes B ; A_{i i}=\mathscr{M}_{i} A \mathscr{M}_{i} .
$$

That is, $\left[\sum E_{i i} \otimes\left(A_{i \mathfrak{i}} \otimes B\right), Q\right] \not \equiv 0$ for all $A, B \in \mathfrak{A}_{n}$ which says that $Q$ is itself a direct sum of the form

$$
Q=P_{1} \otimes P_{2} \otimes \cdots \otimes P_{k},
$$

where $P_{i}$ is an $n_{i} n \times n_{i} n$ matrix. Thus from (2.23),

$$
[A \otimes B, Q]=\sum_{i=1}^{k}\left[A_{i i} \otimes B, P_{i}\right]=\sum_{i=1}^{k} \lambda_{i}\left[A_{i \mathfrak{i}} \otimes B, \mathscr{J}\left(\mathbf{T}_{i}\right)^{\circ}\right]
$$

which says that $P_{i}=\mathscr{J}\left(\mathrm{T}_{i}\right)^{\circ}$ as long as $\lambda_{i} \neq 0$; that is, for $\lambda_{i} \in\left\{\lambda_{1}, \lambda_{2}, \ldots, \lambda_{k-1}, \lambda_{k}\right\}, Q$ is psd if and only if $P_{i}$ is psd for each $i$, which obtains if and only if $\mathscr{J}_{i}\left(\mathbf{T}_{i}\right)$ is psd and the theorem is proved. 
3. Extreme points of $\mathscr{C}_{n_{1}}$. We define the compact, convex set $\mathscr{C}_{n_{1}}$ to be all linear operators $\mathbf{T}$ from $\mathfrak{A}_{n}$ to $\mathfrak{A}_{n}$ such that $\mathbf{T} \in K_{1}$ and $\mathbf{T}$ 2-extends the state $\eta_{1}(\cdot)=[\cdot, P]$ i.e., $\eta_{1}(A \mathbf{T}(B))=\eta_{2}(A \otimes B)$ defines $\eta_{2}$ as a state on $\otimes^{2} \mathfrak{A}$. Similarly, $\mathscr{C}_{\text {tr }}$ is the set of 2-extendable operators $\mathbf{T}$ which preserve positive semidefiniteness and preserve trace.

If $\mathfrak{A}$ is a commutative, selfadjoint (diagonal) algebra, then any $\mathbf{T} \in K_{1} n$-extends any state $\eta$ on $\mathfrak{A}$, for all $n=1,2, \ldots$ In this case, we could apply the result of Ionescu-Tulcea [5] (see also R. R. Phelps [7]) which states (in a more general setting) that the extreme points $\mathbf{T}$ of the convex set $K_{1}$ are exactly the multiplicative operators, i.e., $\mathbf{T}(A B)=\mathbf{T}(A) \mathbf{T}(B)$ for all $A, B \in \mathfrak{A}$.

We characterize the extreme points of $\mathscr{C}_{n_{1}}$, denoted ext $\left(\mathscr{C}_{n_{1}}\right)$, for the simplest, noncommutative case $\mathfrak{A}_{n}=\mathfrak{A}_{2}$, the $2 \times 2$ matrices. We will use some results of [2], in the following.

Lemma 3.1. Suppose $\mathbf{T} \in \mathscr{C}_{n_{1}}$ and $\mathscr{J}(\mathbf{T})$ is positive definite. Then $\mathbf{T} \notin \operatorname{ext}\left(\mathscr{C}_{n_{1}}\right)$.

Proof. Let $\mathscr{J}(\mathbf{T})=\sum_{i=1}^{n^{2}} \lambda_{i} \mathscr{P}\left(X_{i}\right)$, where $\left\{X_{i}\right\}_{i=1}^{n^{2}}$ is o.n. in $L(\bar{H}, H)$ and $\lambda_{i}>0$ for all $i$. Choose $n^{2}$ real numbers $\left\{\varepsilon_{i}\right\}$ so that $\sum \varepsilon_{i} X_{i}^{*} X_{i}=0$, which is possible, since the $n^{2}$ psd operators $\left\{X_{i}^{*} X_{i}\right\}_{i=1}^{n^{2}}$ cannot be linearly independent in $\overline{\mathfrak{A}}=L(\bar{H}, \bar{H})$. Multiplying by a sufficiently small positive constant, we may assume

$$
\max _{i}\left|\varepsilon_{i}\right| \leqq \min _{j} \lambda_{j} .
$$

Hence $\mathscr{J}(\mathbf{U})=\sum\left(\lambda_{i}+\varepsilon_{i}\right) \mathscr{P}\left(X_{i}\right) ; \mathscr{J}(\mathbf{V})=\sum\left(\lambda_{i}-\varepsilon_{i}\right) \mathscr{P}\left(X_{i}\right)$ are psd operators and so $\mathbf{U}, \mathbf{V}:$ psd $\rightarrow$ psd [2, Corollary 2.2]. Moreover, $\mathbf{U}(1)=\mathbf{T}(1)+\varepsilon_{i} X_{i}^{*} X_{i}=1$,

$$
\mathbf{V}(1)=\mathbf{T}(1)-\varepsilon_{i} X_{i}^{*} X_{i}=1 \quad[2, \text { Theorem 2.1] }
$$

We need only show that if $\mathbf{T}$ 2-extends $\eta_{1}$, then so do $\mathbf{U}$ and $\mathbf{V}$. But from (2.14) we see that since $\mathscr{J}(\mathbf{U})^{\circ}, \mathscr{J}(\mathbf{V})^{\circ}$ are psd, they must commute with $P$ in order that $P \mathscr{J}(\mathbf{U})^{\circ}$ and $P \mathscr{J}(\mathbf{V})^{\circ}$ be psd. (That is, in order that $\eta_{2}$ is a state.) But this follows since $\mathscr{J}(\mathbf{U}), \mathscr{J}(\mathbf{V})$ have the same spectral resolution as $\mathscr{J}(\mathbf{T})$, and $\mathscr{J}(\mathbf{T})$ commutes with $P$. The lemma is proved.

At this point, we turn our attention to $\mathfrak{A}_{n}=\mathfrak{A}_{2}$ and present

THeOREM 3.2. Suppose $\eta_{1}(\cdot)=[\cdot, P]$ is a state on $\mathfrak{A}_{2}$. Then $\mathbf{T}$ is extreme in $\mathscr{C}_{\eta}$ if $\mathrm{T}(A)=\omega_{x}(A) P_{z_{1}}+\omega_{y}(A) P_{z_{2}}$, provided the eigenvalues of $P=\lambda_{1} P_{z_{1}}+\lambda_{2} P_{z_{2}}$ are distinct. Moreover, $P_{z_{1}} \cdot P_{z_{2}}=0$.

Proof. Since the eigenvalues of $P$ are presumed to be distinct, we already have (Corollary 2.9) that $\mathbf{T}(A)=\omega_{1}(A) P_{z_{1}}+\omega_{2}(A) P_{z_{2}}$ for states $\omega_{1}, \omega_{2}$ on $\mathfrak{A}_{2} . \mathbf{T}$ is extreme if and only if these states are vector states and this concludes the proof. 
The other case remaining concerns $\eta(\cdot)=\left[\cdot, \frac{1}{2} \cdot 1\right]$ when the eigenvalues of $P$ are equal, i.e., $\eta=\frac{1}{2} \operatorname{tr}(\cdot)$. We may write

$$
\mathscr{J}(\mathbf{T})=\left(\begin{array}{ll}
\mathbf{T}\left(E_{11}\right) & \mathbf{T}\left(E_{21}\right) \\
\mathbf{T}\left(E_{12}\right) & \mathbf{T}\left(E_{22}\right)
\end{array}\right)
$$

where a basis is chosen so that $\mathbf{T}\left(E_{11}\right)$ (hence $\mathbf{T}\left(E_{22}\right)$ ) is diagonal. The operators $E_{i j}$ denote the unit matrices. We state a lemma which was proved by C. Davis [1] for operator algebras.

Lemma 3.3. If $A_{1}^{-1}$ exists, then

$$
\left(\begin{array}{ll}
A_{1} & B \\
B^{*} & A_{2}
\end{array}\right)
$$

is psd if and only if $A_{2}-B^{*} A_{1} B$ is psd.

The matrix representation of $\mathscr{J}(\mathbf{T})$ is given by

$$
\left(\begin{array}{cc}
P & A \\
A^{*} & 1-P
\end{array}\right)
$$

and is therefore psd if and only if

$$
P+A^{*} \frac{1}{P} A \leqq 1
$$

LEMMA 3.4. Let

$$
\mathscr{J}(\mathbf{T})=\left(\begin{array}{cc}
P & A \\
A^{*} & 1-P
\end{array}\right)
$$

if $P^{-1}$ exists. If $\mathrm{T} \in \operatorname{ext}\left(\mathscr{C}_{\mathrm{tr}}\right), A \neq 0$, then $1-P-A^{*}(1 / P) A=\lambda P_{x}$ for some $x, \lambda \geqq 0$.

Proof. Suppose not. $1-P-A^{*}(1 / P) A \neq \lambda P_{x}$ means $\left(1-P-A^{*}(1 / P) A\right)^{-1}$ exists, since we excluded the case where the psd $\left(1-P-A^{*}(1 / P) A\right)$ is either of rank 1 (equal to $\lambda P_{x}$ ), or of rank 0 (equal to $0 \cdot P_{x}$ ). Now it is verified that

$$
P+(A \pm \varepsilon A)^{*} \frac{1}{P}(A \pm \varepsilon A)=P+A^{*} \frac{1}{P} A+|\varepsilon|^{2} A^{*} \frac{1}{P} A
$$

if $\bar{\varepsilon}=-\varepsilon$. Now $P+A^{*}(1 / P) A$ is psd $\leqq 1$ by (3.3), and we have

$$
1-P-A^{*} \frac{1}{P} A=Q, \mathrm{psd}
$$

which is nonsingular by hypothesis. Thus

$$
\min _{x}\left[Q, P_{x}\right]=\sigma>0,
$$

by compactness of the unit sphere in $\mathscr{H}_{2}$, and

$$
\max _{y}\left[A^{*} \frac{1}{P} A, P_{y}\right]=\Sigma \text {. }
$$


Setting $|\varepsilon|^{2} \leqq \sigma / \Sigma$, we have, for all $x$,

$$
|\varepsilon|^{2}\left[A^{*} \frac{1}{P} A, P_{x}\right]<|\varepsilon|^{2} \Sigma \leqq \sigma=\min _{z}\left[Q, P_{z}\right] \leqq\left[Q, P_{x}\right]
$$

Thus, $|\varepsilon|^{2} A^{*}(1 / P) A \leqq 1-P-A^{*}(1 / P) A$ if and only if

which says that

$$
|\varepsilon|^{2} A^{*} \frac{1}{P} A+P+A^{*} \frac{1}{P} A \leqq 1,
$$

$$
\left(\begin{array}{cc}
P & A+\varepsilon A \\
A^{*}-\varepsilon A^{*} & 1-P
\end{array}\right) \text { and }\left(\begin{array}{cc}
P & A-\varepsilon A \\
A^{*}+\varepsilon A^{*} & 1-P
\end{array}\right) \text { are psd. }
$$

Hence $\mathscr{J}(\mathbf{T})$, being the average of these two matrices, is not extreme so $\mathbf{T} \notin \operatorname{ext}\left(\mathscr{C}_{\text {tr }}\right)$.

Lemma 3.5. $A=0, P^{-1}$ exists. $\mathrm{T} \in \operatorname{ext}\left(\mathscr{C}_{\mathrm{tr}}\right)$ then $\mathrm{T}(\cdot)=\omega_{x}(\cdot) 1$ for some vector state $\omega_{x}$.

Proof.

$$
\mathscr{J}(\mathbf{T})=\left(\begin{array}{cc}
P & 0 \\
0 & 1-P
\end{array}\right)=\left(\begin{array}{cccc}
\lambda & 0 & 0 & 0 \\
0 & \mu & 0 & 0 \\
0 & 0 & 1-\lambda & 0 \\
0 & 0 & 0 & 1-\mu
\end{array}\right)
$$

assuming $P=\mathbf{T}\left(E_{11}\right)$ is diagonal. If $\mathbf{T}$ is extreme, Lemma 3.1 says that $\mathscr{J}(\mathbf{T})$ is singular. $P^{-1}$ exists so that

$$
P=\left(\begin{array}{ll}
1 & 0 \\
0 & \mu
\end{array}\right) \text { or }\left(\begin{array}{ll}
\lambda & 0 \\
0 & 1
\end{array}\right),
$$

which is not extremal unless $\lambda$ or $\mu=1$. Thus $\mathbf{T}\left(E_{11}\right)=1$ or $\mathbf{T}(\cdot)=\omega_{x}(\cdot) 1$.

Lemma 3.6. Suppose $P^{-1}$ and $(1-P)^{-1}$ do not exist. The extremal $\mathbf{T} \in \mathscr{C}_{\mathrm{tr}}$ are the antimultiplicative operators $A \rightarrow U^{*} A^{t} U$, or the multiplicative $A \rightarrow U^{*} A U$ for $U^{-1}=U^{*}$, where $A^{t}$ denotes the transpose of $A$.

Proof.

$$
\mathscr{J}(\mathbf{T})=\left(\begin{array}{llll}
1 & 0 & 0 & e^{-i \theta} \\
0 & 0 & 0 & 0 \\
0 & 0 & 0 & 0 \\
e^{i \theta} & 0 & 0 & 1
\end{array}\right)
$$

which is the only possibility for this case, assuming

$$
P=\mathbf{T}\left(E_{11}\right)=\left(\begin{array}{ll}
1 & 0 \\
0 & 0
\end{array}\right),
$$


i.e., the corner matrices $\mathbf{T}\left(E_{i j}\right)=\left(f_{j} \times f_{i}\right)$ and $\mathbf{T}$ is antimultiplicative. If

$$
P=\left(\begin{array}{ll}
0 & 0 \\
0 & 1
\end{array}\right)
$$

is assumed, then $\mathbf{T}$ is multiplicative.

Lemmas 3.5 and 3.6 have been proved, where nonvoid examples exist. However, no nonvoid example exists for Lemma 3.4. Suppose, in fact, that $P^{-1}$ exists, $A \neq 0$ and $P+A^{*}(1 / P) A=1-\lambda P_{x}, 0 \leqq \lambda \leqq 1$. If $P^{-1}$ and $(1-P)^{-1}$ exist, then the trace of one of the operators $P$ or $1-P$ exceeds unity; say $\operatorname{tr}(P)>1$. Thus

$$
1<\operatorname{tr}\left(P+A^{*} \frac{1}{P} A\right)=\operatorname{tr}\left(1-\lambda P_{x}\right)=1-\lambda \leqq 1 .
$$

The first inequality is strict since $A \neq 0$, and a contradiction ensues.

If $P^{-1}$ exists and $1-P$ has no inverse, then $P$ may be represented by

$$
\left(\begin{array}{ll}
1 & 0 \\
0 & \lambda
\end{array}\right)
$$

Again $\operatorname{tr}(P)>1$ and the argument above yields the contradiction. We have proved

THEOREM 3.7. The extreme points of $\mathscr{C}_{\eta}$, where $\eta=\frac{1}{2}$ tr are the operators

$$
\mathbf{T}_{x}: A \rightarrow \omega_{x}(A) 1, \quad \mathbf{T}_{U}: A \rightarrow U^{*} A U \text { or } \mathbf{T}_{U}^{\prime} A \rightarrow U^{*} A^{t} U
$$

where $U^{*}=U^{-1}$, and $A^{t}$ represents the transpose of $A$. The extreme points of $\mathscr{C}_{\eta}$ where $\eta(\cdot)=[\cdot, P], P=\lambda_{1} P_{z_{1}}+\lambda_{2} P_{z_{2}}, \lambda_{1} \neq \lambda_{2}$ are the operators

$$
\mathbf{T}_{x y}: A \rightarrow \omega_{x}(A) P_{z_{1}}+\omega_{y}(A) P_{z_{2}},
$$

where $\omega_{x}, \omega_{y}$ are vector states and $P_{z_{1}} \cdot P_{z_{2}}=0$.

\section{REFERENCES}

1. C. Davis, A device for studying Hausdorff moments, Trans. Amer. Math. Soc. 87 (1958), 144-158.

2. J. de Pillis, Linear operators which preserve hermitian and positive semidefinite transformations, (to appear).

3. J. L. Doob, Stochastic processes, Wiley, New York, 1953.

4. H. A. Dye, The Radon-Nikodym Theorem for finite rings of operators, Trans. Amer. Math. Soc. 72 (1952), 243-280.

5. A. and C. Ionescu-Tulcea, On the lifting property (I), J. Math. Anal. Appl. 3 (1961), 537-546.

6. M. Naimark, Normed rings, Noordhoff, Groningen, 1964.

7. R. R. Phelps, Extreme positive operators and homomorphisms, Trans. Amer. Math. Soc. 108 (1963), 265-274.

8. I. E. Segal, A noncommutative extension of abstract integration, Ann. of Math. 57 (1953), 401-457.

UNIVERSITY OF CALIFORNIA,

Riverside, California 